\title{
Surgical instrumentation: the true cost of instrument trays and a potential strategy for optimization
}

\author{
Julie M. Mhlaba ${ }^{1,2}$, Emily W. Stockert ${ }^{1,2}$, Martin Coronel ${ }^{2}$, Alexander J. Langerman ${ }^{* 2,3}$ \\ ${ }^{1}$ Pritzker School of Medicine, University of Chicago, Chicago, United States \\ ${ }^{2}$ Department of Surgery, Operative Performance Research Institute, University of Chicago, Chicago, United States \\ ${ }^{3}$ Department of Surgery, Section of Otolaryngology-Head and Neck Surgery, University of Chicago, Chicago, United States
}

Received: June 2, 2015

DOI: $10.5430 /$ jha.v4n6p82
Accepted: August 6, 2015

Online Published: September 23, 2015

URL: http://dx.doi.org/10.5430/jha.v4n6p82

\begin{abstract}
Objective: Operating rooms (OR) generate a large portion of hospital revenue and waste. Consequently, improving efficiency and reducing waste is a high priority. Our objective was to quantify waste associated with opened but unused instruments from trays and to compare this with the cost of individually wrapping instruments.

Methods: Data was collected from June to November of 2013 in a 550-bed hospital in the United States. We recorded the instrument usage of two commonly-used trays for ten cases each. The time to decontaminate and reassemble instrument trays and peel packs was measured, and the cost to reprocess one instrument was calculated.

Results: Average utilization was 14\% for the Plastic Soft Tissue Tray and 29\% for the Major Laparotomy Tray. Of 98 instruments in the Plastics tray $(n=10), 0 \%$ was used in all cases observed and 59\% were used in no observed cases. Of 110 instruments in the Major Tray $(\mathrm{n}=10), 0 \%$ was used in all cases observed and $25 \%$ were used in no observed cases. Average cost to reprocess one instrument was $\$ 0.34-\$ 0.47$ in a tray and \$0.81-\$0.84 in a peel pack, or individually-wrapped instrument.

Conclusions: We estimate that the cost of peel packing an instrument is roughly two times the cost of tray packing. Therefore, it becomes more cost effective from a processing standpoint to package an instrument in a peel pack when there is less than a $42 \%-56 \%$ probability of use depending on instrument type. This study demonstrates an opportunity for reorganization of instrument delivery that could result in a significant cost-savings and waste reduction.
\end{abstract}

Key Words: Operating rooms, Surgical instruments, Cost benefit analysis, Efficiency

\section{INTRODUCTION}

In the current era of increasing focus on health expenditures, opportunities for cost saving measures in the operating rooms (OR) must be identified and maximized. ${ }^{[1]}$ In addition, with the recent push towards sustainability in the healthcare system, ${ }^{[2,3]}$ the OR has been identified as a target for environmentally conscious interventions. ${ }^{[4,5]}$ One largely unexplored area over which surgeons may have direct influence is surgical instrumentation.

Previous research from our group found that instrument uti- lization, or the percentage of instruments used from a given tray opened for a surgical case, was low for multiple trays across four surgical specialties $(13 \%-21.9 \%)$. When an instrument tray is opened for a surgical case, all instruments in the tray are "exposed" and must be decontaminated, reassembled and sterilized, independent of whether or not they were used in the case. After factoring in labor, instrument depreciation and operating costs for sterile processing, we estimated that it costs up to $\$ 0.51$ to clean and re-package each exposed instrument. ${ }^{[6]}$ These unused but exposed instruments represent systematic waste and potential targets

*Correspondence: Alexander J. Langerman; Email: Alexander.Langerman@ vanderbilt.edu; Address: Vanderbilt Department of Otolaryngology, 7290 Medical Center East, South Tower, Nashville, TN 37232, United States. 
for cost savings.

Reducing the waste of unused instruments must be balanced against ensuring that less-frequently used but critical instruments remain readily available. Farrokhi et al. used a Lean framework to determine the minimum amount of instruments necessary to perform minimally invasive spine surgeries safely and efficiently. Following their intervention, they found a $70 \%-80 \%$ reduction in the number of instruments in the trays studied. ${ }^{[7]}$ This findings has been replicated across specialties and institutions, further strengthening the argument that instrument reduction is both feasible and can substantially reduce waste. ${ }^{[8]}$

The goals of the present study were to examine the patterns of individual instrument use for two surgical services. Although we knew that overall utilization was low, we had not determined if the same small group of instruments was being repeatedly used, or whether different surgeons used separate, distinct groups of instruments from the same trays. Additionally, we sought to examine if the "used" instruments were used in every case or in some cases.

The latter distinction is important, because group tray sterilization is only one method for providing instruments to the operating room. An alternative method involves individually wrapping ("peel packing") instruments. Peel-packed instruments have consistently proved to be a safe an effective method for instrument storage, with one study showing that autoclaved packages in double-wrapped plastic can be safely stored up to 96 weeks. ${ }^{[9,10]}$ While peel packing might not be a cost-effective method for consistently used instruments, it could provide a potential alternative to infrequently used instruments. This would save the infrequently used instruments from constant, unnecessary reprocessing, which carries labor and equipment costs and contributes to instrument depreciation. ${ }^{[6]}$ If a peel-packed instrument is not opened for a given procedure, it can be made available for another procedure without being sterilized and repackaged. In addition, using peel packs instead of trays for the storage of selected instruments may lead to a reduction in tray weight, an important consideration for employee safety. ${ }^{[11]}$ Thus, as a final component to this study, we sought to estimate the cost of peel packing in order to determine the inflection point at which it becomes more cost-effective to peel pack rather than tray pack an instrument.

\section{METHODS}

\subsection{Study design}

This was a single-site, observational study conducted on the surgical instrumentation used at a large academic medical center. Two commonly used surgical instrument trays were identified as potential candidates for reduction through hosPublished by Sciedu Press pital records on frequency of use and interviews with OR staff members: "Major Laparotomy Tray" and "Plastic Soft Tissue Tray". Hospital records demonstrated that these tray types were processed at a high volume (each $>1,000$ times per year). In addition, interviews with specialty managers and central sterile processing (CSP) employees led to the conclusion that these trays exhibited potential for instrument reduction. These are "general" trays, and thus are used by multiple surgeons for multiple procedures. All surgeons, nurses, operating room personnel, and central sterile processing staff involved in this study were educated on the data being collected and the purpose of the research. This research was approved by the University of Chicago Institutional Review Board.

\subsection{Data collection - operating room}

Surgeons from the Plastic and General surgical services were contacted prior to this study for willingness to have an observer in their operating room. An observer entered the OR prior to patient entry to obtain oral consent from the circulating nurse and the surgical technician. At the end of operative time, all instruments used from the tray of interest were reported to the observer by the surgical technician. No patient data was collected. Data was collected on routine surgical procedures requiring the two instrument trays from June through November of 2013.

\subsection{Data collection - central sterile processing}

For the purpose of identifying differences between instrument types with regards to processing labor time, we categorized instruments as follows: "open" - requiring a CSP employee to manually open the instrument prior to processing (e.g. a clamp or scissors); "closed" - no opening required (e.g. a retractor); and "lumen" - instruments that require irrigation for cleaning (e.g. a suction). Using a calibrated stopwatch, an investigator recorded the time to decontaminate each instrument type and assemble it within an instrument tray.

In addition, we investigated the processing of peel-packed instruments in CSP. Data was collected on the following aspects of peel-pack assembly: instrument type, time to peel-pack individual instruments, and type and dimensions of peel-packing material used. The cost of peel-packing material and operating costs was provided by hospital administration. Cost calculations for both tray and peel-packed instruments included equipment maintenance and repair as well as instrument repair and supplies (indicators, detergents etc.) and were distributed equally across instrument types.

\subsection{Data analysis}

Instrument utilization for each tray was calculated using the following equation: 


$$
\text { Instrument utilization }=\frac{\text { InstrumentCount }_{U S E D}}{\text { InstrumentCount }_{T O T A L}}
$$

Time to decontaminate and reassemble for individual instruments by type was calculated by dividing the total time per instrument category by the number of instruments in each

\section{RESUlts}

\subsection{Tray utilization}

Twenty surgical cases were observed, 10 each for the Plastic Soft Tissue and Major Laparotomy trays. Average instrument utilization for the 20 trays observed was $22 \%$ with a category. The cost of processing per instrument packed in a tray was calculated by the following equation:

Table 1. Distribution of instruments utilized by tray type in the study hospital, USA, 2013

\begin{tabular}{|c|c|c|c|c|c|c|c|c|c|c|c|c|}
\hline Tray & Average & $S D$ & 1 & 2 & 3 & 4 & 5 & 6 & 7 & 8 & 9 & 10 \\
\hline PST & $29 \%$ & $\pm 3 \%$ & $14 \%$ & $10 \%$ & $13 \%$ & $9 \%$ & $12 \%$ & $16 \%$ & $15 \%$ & $17 \%$ & $17 \%$ & $18 \%$ \\
\hline ML & $14 \%$ & $\pm 10 \%$ & $31 \%$ & $33 \%$ & $27 \%$ & $16 \%$ & $37 \%$ & $33 \%$ & $35 \%$ & $43 \%$ & $9 \%$ & $28 \%$ \\
\hline
\end{tabular}

Note. PST = Plastic Soft Tissue tray; ML = Major Laparotomy tray

We observed three plastic surgeons performing five types of procedures that utilized the Plastic Soft Tissue Tray. Of the 98 instruments in this tray, $0(0 \%)$ instruments were used in all cases observed, $41(40 \%)$ instruments were used in some of the cases observed and $58(59 \%)$ instruments were used in none of the cases observed.

We observed four general surgeons performing five types of procedures that utilized the Major Laparotomy Tray. Of the 110 instruments in this tray, $0(0 \%)$ instruments were used in all cases observed, $83(75 \%)$ instruments were used in some of the cases observed and $27(25 \%)$ instruments were used in none of the cases observed (see Figure 1).

\subsection{Central sterile processing}

A total of sixteen trays of varying types (range of 31-188 instruments per tray, 1,525 instruments total) were observed being decontaminated and a total of nine trays of varying types (range of 6-128 instruments per tray, 618 instruments standard deviation of $\pm 10 \%$ and a range of $9 \%-43 \%$ instrument utilization). Average tray-specific instrument utilization was $14 \%$ for Plastic Soft Tissue with a standard deviation of $\pm 3 \%$ and a range of $9 \%-18 \%$ and $29 \%$ for Major Laparotomy with a standard deviation of $\pm 10 \%$ and a range of $9 \%-43 \%$ (see Table 3 ). total) were observed being assembled in CSP. The average time to decontaminate one "closed", "open" and "lumen" instrument was 2.7, 3.2 and 7.2 seconds, respectively. Decontamination took an average of 5.0, 11.7 and 22.3 seconds per instrument type, respectively.

Ten instruments were observed during peel packing. The average time for peel packing per instrument was 75.6 seconds. Processing labor time for peel pack assembly was consistent across instrument types (closed, open, lumen). The average cost of peel-packing material per instrument was $\$ 0.05$.

In order to compare the cost of packing instruments in trays versus peel pack, we estimated the total cost of reprocessing a single instrument using each method. Total estimates took into account direct costs (labor and depreciation) as well as indirect costs (utilities and repair) as shown in Table 2.

Direct costs from labor were calculated using the equation below:

Total labor cost per instrument $=$ Employee wage per second $\times$ Total time to clean and reassemble an instrument

According to calculations from Stockert et al., indirect costs amount to $\$ 0.23$ per instrument, which includes the operating cost of detergent, biologic and quality checks, maintenance and repair. The average cost of per use instrument depreci- ation is estimated at $\$ 0.06$. These estimations were calculated assuming CSP operates at $80 \%$ capacity, as shown in Table 3. ${ }^{[6]}$ 

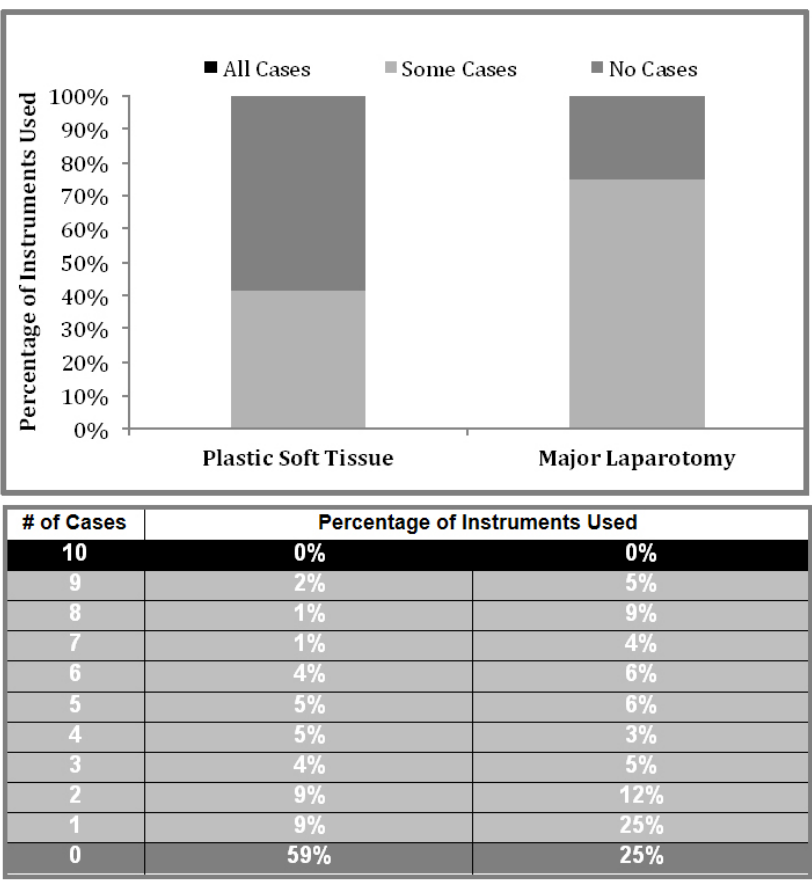

Figure 1. Percentage of instruments used in some (1-9) cases and zero cases is graphed by tray type

None of the instruments were used in all (10) cases observed. The percentage of instruments used is broken down further below by the exact number of cases used.
According to these calculations, we estimate that the cost of tray packing a single instrument is $\$ 0.34$ for a "closed" instrument, $\$ 0.38$ for an "open" instrument and $\$ 0.47$ for a "lumen" instrument. Additionally, we estimate that the cost of peel packing a single instrument is $\$ 0.81$ for a "closed" or "open" instrument and $\$ 0.84$ for a "lumen" instrument. Accordingly, the cost of peel packing is 2.4 times the cost of tray packing a "closed" instrument, 2.1 times the cost of tray packing an "open" instrument and 1.8 times the cost of tray packing a "lumen" instrument (see Table 3). However, a real cost comparison between peel-packed instruments and trays must take into account the cost of unnecessarily processing of instruments. For example, if an instrument in a tray is used only once in every ten times the tray is opened and reprocessed, then the real cost of that instrument per use is ten times the reprocessing cost. In other words, a "closed" instrument with a $10 \%$ utilization that costs $\$ 0.34$ per reprocessing would equate to a real cost of $\$ 3.40$ per use if kept in a tray. However, if that same "closed" instrument was placed in a peel pack, it should only be opened for intended use; the $\$ 0.81$ cost of peel packing that instrument equates to $\$ 0.81$ per use, almost one-fourth the actual cost of the same instrument packaged in a tray.

Table 2. Labor cost varies by instrument type and packaging method, USA, 2013

\begin{tabular}{llllll}
\hline Packaging & Instrument Type & Time to Decontaminate & Time to Assemble & Total Time & Total Labor Cost \\
\hline \multirow{3}{*}{ Tray } & Closed & $2.7 \mathrm{~s}$ & $5.0 \mathrm{~s}$ & $7.7 \mathrm{~s}$ & $7.7 \times \$ 0.006=\$ 0.05$ \\
& Open & $3.2 \mathrm{~s}$ & $11.7 \mathrm{~s}$ & $14.9 \mathrm{~s}$ & $14.9 \times \$ 0.006=\$ 0.09$ \\
& Lumen & $7.2 \mathrm{~s}$ & $22.3 \mathrm{~s}$ & $29.5 \mathrm{~s}$ & $29.5 \times \$ 0.006=\$ 0.18$ \\
\multirow{3}{*}{ Peel-Pack } & Closed & $2.7 \mathrm{~s}$ & $75.6 \mathrm{~s}$ & $78.3 \mathrm{~s}$ & $78.3 \times \$ 0.006=\$ 0.47$ \\
& Open & $3.2 \mathrm{~s}$ & $75.6 \mathrm{~s}$ & $78.8 \mathrm{~s}$ & $78.8 \times \$ 0.006=\$ 0.47$ \\
& Lumen & $7.2 \mathrm{~s}$ & $75.6 \mathrm{~s}$ & $82.8 \mathrm{~s}$ & $82.8 \times \$ 0.006=\$ 0.50$ \\
\hline
\end{tabular}

Note. Time to decontaminate and assemble measured using a calibrated stopwatch; Total labor cost calculated using equation (3); Trays: $\mathrm{n}=16$; Peel-pack: $\mathrm{n}=10$

Table 3. Total cost for processing varies by instrument type and packaging method, USA, 2013

\begin{tabular}{lllllll}
\hline Packaging & Instrument Type & Labor & Depreciation & Utilities \& Repair & Peel-Packing & Total Cost \\
\hline \multirow{3}{*}{ Tray } & Closed & $\$ 0.05$ & $\$ 0.06$ & $\$ 0.23$ & $\$ 0.34$ & $\$ 0.38$ \\
& Open & $\$ 0.09$ & $\$ 0.06$ & $\$ 0.23$ & $\$ 0.47$ & $\$ 0.81$ \\
& Lumen & $\$ 0.18$ & $\$ 0.06$ & $\$ 0.23$ & $\$ 0.05$ & $\$ 0.81$ \\
& Closed & $\$ 0.47$ & $\$ 0.06$ & $\$ 0.23$ & $\$ 0.05$ & $\$ 0.84$ \\
\hline
\end{tabular}

Note. Labor costs calculated using equation (3); Costs estimates for depreciation, utilities \& repair and materials provided by hospital administration; Trays: $\mathrm{n}=16$; Peel-pack: $\mathrm{n}=10$

\section{Discussion}

Our data show that the use of instruments in trays is heterogenous. Some instruments are used frequently, across many

Published by Sciedu Press surgeons and types of cases, and others rarely or not at all. For example, the Adson Forceps, an instrument commonly used for grasping tissue or gauze dressing, were used in nine 
of ten of the Majority Laparotomy cases. Alternatively, the Fine, J-Curve Forceps were used in only one of the ten Major Laparotomy cases. We feel that, with data on enough cases, the probability of use can be estimated for each instrument.

Overall utilization of the trays was low, with a substantial percentage (25\%-59\%) of instruments going unused. This data supports previous findings of low instrument usage ${ }^{[6,7]}$ and demonstrates that these numbers hold when considering instruments individually. In addition, this data suggests that specific, low-utilization instruments may be identified that may be amenable to peel packing.

Based on our cost estimates, we can resolve that the inflection point at which it becomes more cost effective to package a "closed" instrument in a peel pack versus an instrument tray is when that instrument is actually used in less than $42 \%$ of the cases for which it is available - in other words, an instrument that has less than a $42 \%$ chance of use. The inflection point for which it is more cost-effective to peel pack an "open" instrument is when an instrument has less than a $47 \%$ chance of use. Finally, the inflection point for a "lumen" instrument is when that instrument has less than a $56 \%$ chance of use.

Below these inflection points, the cost of unnecessarily reprocessing tray packed instruments exceeds the cost of peel packing the same instrument. Thus, while the absolute cost of peel packing a given instrument may be more expensive than tray packing the same instrument, the real cost of each method must take into account the context in which that instrument is used. The packaging of these uncommonly used instruments in trays leads to avoidable cost and operative inefficiencies. As described, each reprocessing requires employee labor, processing supplies, and equipment maintenance. Peel-packed instruments require this reprocessing only after they are used in a case and are then able to be stored compactly until needed.

In addition, a significant portion of instruments in trays was not used in any observed cases (59\% for Plastic Soft Tissue, $25 \%$ for Major Laparotomy). At an average reprocessing cost of $\$ 0.40$ per instrument, removal of 58 unused instruments in the Plastic Soft Tissue Tray would save $\$ 23$ of waste per tray use. As this tray was used roughly 1,300 times in 2013 , this would save approximately $\$ 29,900$ per year for just one type of tray. This estimate suggests that reduction of instrument trays could provide a significant opportunity for cost-savings, and indeed we have undertaken this reduction based on our data and surgeon feedback.

We focused on "general", rather than case- or surgeonspecific trays, to examine utilization when multiple surgeons use a single tray - their individual preferences and/or case requirements resulted in different instruments being used. General trays are advantageous because they are a form of pooled inventory ${ }^{[12]}$ - by serving multiple surgeons and cases the number of spare trays kept as "safety stock" can be minimized compared to when specialized trays are used for each surgeon. However, the value of inventory pooling in settings of highly variable demand (such as with instruments in the two trays we investigated) may be diminished and is worthy of further investigation. Regardless, it is intuitive that keeping very infrequently (or never) used instruments in general trays is a source of waste.

The purpose of this study was to demonstrate that variability in individual instrument usage exists within trays and to illustrate that understanding this feature could serve as an opportunity for tray reduction. A comprehensive and standardized process should be used to gather further data and ensure a smooth transition, such as $5 \mathrm{~s}$ (sort, simplify, sweep, standardize and self-discipline). ${ }^{[7]}$ Furthermore, the use of specialized software to predict sterility costs is already in development and should be capitalized upon. ${ }^{[13]}$

In addition, this study presents an opportunity to address sustainable practices in healthcare. It is estimated that operating rooms generate from $20 \%-33 \%$ of total hospital waste. ${ }^{[5,14]}$ Previously cited areas for waste reduction include instituting reusable surgical linens, finding alternatives to incineration and utilizing anesthetic collection services. ${ }^{[5]}$ The removal of unnecessary surgical instruments from instrument trays serves as an additional opportunity for waste reduction through the lessening of water and cleaning supplies required for reprocessing. This must be balanced against the waste (albeit recyclable) generated by using disposable plastic-andpaper peel packaging.

Any effort to reduce instrument tray size must be balanced against instrument availability for unexpected scenarios, both to protect patient safety and avoid potential delays. Peel packing is a feasible option for keeping instruments sterile yet available across multiple cases. As detailed above, we determined that the cost-based inflection point was at a $42 \%-56 \%$ probability of use (depending on instrument type), below which it is less expensive to peel pack an instrument. The peel-packed instruments can then be delivered to the OR, with the tray, and, if unopened, returned to CSP and prepared for the next cases without undergoing decontamination and assembly.

In addition, the peel packing of instruments need not be restricted to those instruments rarely used. In fact, instruments that are commonly used are also good candidates for peel packing. In our current system, in order to intra- 
operatively replace certain instruments that are contaminated or defective, the entire tray containing that instrument must be requested, opened and, after the procedure, decontaminated and reassembled. At times, a large tray is opened for one critical instrument. ${ }^{[6]}$ Peel packing critical instruments would offer a cost-effective and simple solution to this issue, as contaminated or defective instruments could be replaced singly. In addition, a small number of instruments that are commonly used together (e.g. skin closure) might be peel packed as a group to avoid the need for a full tray, as is often done with disposable sets.

This may even be taken a step further to modular trays trays with individual compartments that travel together but are independently sterile. Only the compartments with the instruments to be used would be opened. Planning a modular tray would require an analysis of the "relatedness" of instruments (the likelihood of their being used together). We did not explore modular trays directly in this study, but the all-or-none principle of peel packs applies, with an alteration of the calculus to include the upfront investment in a durable tray and eliminate the peel packaging.

While peel packing is likely to result in cost-savings, it is important to consider logistical costs of tracking packs, ensuring that multiple independent packs are delivered to the OR and the "hassle factor" associated with the opening of a peel-pack (or modular tray) intra-operatively. Radio-frequency identification (RFID) has made such logistics easier. ${ }^{[15]}$ Future work should focus on assessing the impact on tray reduction by employing temporary peel packs and collecting additional data on the usage of the instruments that are considered below the point of value. The logistical costs of managing multiple packs would almost certainly reduce the inflection point to a lower likelihood of utilization.

There are limitations to this study. Data was collected at a single-institution on a select group of trays. As the organization of supplies, instruments and staffing in ORs varies between hospitals, this data may have to be adapted to local practices. In addition, larger sample sizes may be needed to ensure usage patterns and the operating surgeons must be consulted before tray reduction strategies can be implemented. Nevertheless, we feel that the concepts addressed in this study are applicable to surgical administrations nationwide. Institutions would benefit from evaluating the efficiency of their surgical operations, including an examination of their surgical instrument processing. Both cost-savings and sustainable practices can be more readily achieved when the status quo is consistently reassessed; this study aids in the process of doing so.

\section{CONClusions}

This study demonstrates an opportunity for a reorganization of instrument delivery to the OR that could result in a significant cost-savings. The use of peel packing, both for seldom-used instrument and for commonly-used or related instruments, has the potential to result in a reduction of unnecessary reprocessing and operating costs.

\section{REFERENCES}

[1] National Center for Health Statistics. Health, United States, 2009, with chartbook on trends in the health of Americans. 2007.

[2] Harris N, Pisa L, Talioaga S, et al. Hospitals going green: a holistic view of the issue and the critical role of the nurse leader. Holist Nurs Pract. 2009 Apr; 23(2): 101-11. PMid: 19258852. http://dx.doi.org/10.1097/HNP.0b013e3181a110fe

[3] Kaplan S, Sadler B, Little K, et al. Can sustainable hospitals help bend the health care cost curve? Issue Brief Commonw Fund. 2012 Nov; 29: 1-14.

[4] Kwakye G, Brat GA, Makary MA. Green surgical practices for health care. Arch Surg Chic Ill 1960. 2011 Feb; 146(2): 131-6.

[5] Kagoma Y, Stall N, Rubinstein E, et al. People, planet and profits: the case for greening operating rooms. Cmaj Can Med Assoc J J Assoc Medicale Can. 2012 Nov 20; 184(17): 1905-11. PMid: 22664760. http://dx.doi.org/10.1503/cmaj.112139

[6] Stockert EW, Langerman AJ. Assessing the Magnitude and Costs of Intraoperative Inefficiencies Attributable to Surgical Instrument Trays. Journal of the American College of Surgeons. 2014 Oct; 219(4): 646-655.

[7] Farrokhi FR, Gunther M, Williams B, et al. Application of Lean Methodology for Improved Quality and Efficiency in Operating
Room Instrument Availability. J Healthc Qual Off Publ Natl Assoc Healthc Qual. 2015 Mar 13.

[8] Chin C, Sowerby L, John-Baptiste A, et al. Reducing otolaryngology surgical inefficiency via assessment of tray redundancy. J Otolaryngol Head Neck Surg. 2014 Dec 3; 43(1): 46. PMid: 25466550. http://dx.doi.org/10.1186/s40463-014-0046-2

[9] Barrier properties and cost implications of a single versus a double wrap for storing sterile instrument packs. - PubMed - NCBI [Internet] [cited 2015 Jun 28]. Available from: http://www.ncbi.nlm.nih .gov/pubmed/16061141

[10] Long-term storage of small surgical instruments in autoclaved packages [cited 2015 Jun 28]. Available from: http://www.ncbi .nlm.nih.gov/pubmed/?term=Long-term+storage+of+sma $11+$ surgical+instruments+in+autoclaved+packages

[11] Understanding current steam sterilization recommendations and guidelines. EBSCOhost [Internet]. [cited 2015 Jun 28]. Available from: http://web.a.ebscohost.com.proxy.uchicago.edu /ehost/pdfviewer/pdfviewer?sid=c8865c33-36eb-478 $c-83 c f-98 f a 7 f 5 c b 72 a \% 40$ sessionmgr $4002 \& v i d=1 \& h i d=421$ 4

[12] Eppen GD. Note-Effects of Centralization on Expected Costs in a Multi-Location Newsboy Problem. Manag Sci [Internet]. 1979 
May; 25(5): 498-501. http://dx.doi.org/10.1287/mnsc.25 .5 .498

[13] article-t-doc-t-docinzentralsterilization-sal2355-01-us.pdf [Internet]. [cited 2015 Jun 28]. Available from: http://www.getinge.com/ files/HC/products/sterile-supply-management-trace ability-solutions/complete-sterile-supply-managem ent-traceability-solutions/t-doc-2000/case $\% 20$ studi es/article-in-zentral-sterilization-2010/article-t -doc-t-docinzentralsterilization-sal2355-01-us.pdf

[14] Tieszen ME, Gruenberg JC. A quantitative, qualitative, and critical assessment of surgical waste. Surgeons venture through the trash can. JAMA. 1992 May; 267(20): 2765-8. PMid: 1578596. http://dx.doi.org/10.1001/jama.1992.03480200073027

[15] Greene J. Gaining efficiency with instrument tracking. OR Manager. 2004 Mar; 20(3): 17-9, 21-2. 